\title{
A new term for a better distinction? A view from the higher end of the proficiency scale
}

Keywords: high proficiency heritage speakers, simultaneous bilinguals, bilingualism in Europe, phonetic and phonological skills

Tanja Kupisch: University of Hamburg. E-mail: tanja.kupisch@uni-hamburg.de

\section{Introduction}

Benmamoun, Montrul and Polinsky (hereafter: BMP) provide an impressive portfolio of research featuring predominantly the language of second generation bilinguals in their weaker or "heritage" language. Section 2 starts with a general introduction to the population of heritage speakers (hereafter: HS), including how to identify them and measure their proficiency in the heritage language. Section 3 provides a general overview of HS grammars, making reference to their sound systems, morphology, syntax and semantics. Section 4 highlights some linguistic features typical of heritage speakers, such as problems with interface phenomena. Section 5 discusses factors that may be crucial in shaping HS grammars.

In what follows, I take up four aspects of the paper, which are most relevant from my point of view given my background. I start by repeating some of the descriptions of HS given by BMP. I then turn to the question whether the concept of HS is indeed poorly understood outside of the U.S., because under the given description of HS, as I understand it, there are many relevant studies outside of the U.S. that the authors did not take into account. What is more, had they considered them, a different picture of HS may have emerged. While drawing mainly on data from HS at the lower end of the proficiency scale, BMP suggests that HS are more often than not subject to "incomplete acquisition" or attrition ("bilingual speakers of an ethnic or immigrant minority language, whose first language often does not reach native-like attainment in adulthood."). In section 4 I argue that one could come to different conclusions based on research in Europe. Section 5 shows that simultaneous bilinguals, despite having majority language input from the earliest possible age, do not necessarily end up with more divergent grammars than successive bilinguals. In section 6 I summarize research showing that 
accent is the most vulnerable aspect of language in HS at the higher end of the proficiency scale.

\section{Who is a heritage speaker?}

Because the relevance of this commentary depends on how one defines HS, I begin by repeating what, according to BMP, characterizes them. BMP provide the following descriptions, here numbered (1)-(4) [emphasis mine]. As I argue below, when taken together, these do not seem to be completely consistent, which is why I do not treat them as definitions.

(1) "Heritage speakers [...] are bilingual speakers of an ethnic or immigrant minority language, whose first language often does not reach native-like attainment in adulthood" (p. 129).

(2) "The term heritage speaker typically refers to second generation immigrants, the children of the original immigrants, who live in a bilingual/multilingual environment from an early age. Heritage speakers have as their dominant language the language of the host country." (p. 132)

(3) " $[\mathrm{A}]$ heritage speaker is an early bilingual who grew up hearing (and speaking) the heritage language (L1) and the majority language (L2) either simultaneously or sequentially in early childhood (that is, roughly up to age $5[\ldots]$ ), but for whom L2 became the primary language at some point during childhood (at, around, or after the onset of schooling)” (p. 133).

(4) "[I]ndividuals raised in homes where a language other than English is spoken and who are to some degree bilingual in English and the heritage language." According to the authors, "[t]he crucial criterion is that the heritage language was first in the order of acquisition but did not develop fully at age appropriate levels because of the individual's switch to the societally-dominant language" (p. 133).

First, notice that, according to (4), incomplete acquisition seems to define heritage speakers. In other words, those who develop at age-appropriate levels will not count among the group of HS. It is stated further that HS will not develop linguistically at age-appropriate levels because of their switch to the societally dominant language. This statement could easily be (mis-)taken to imply that mastering two languages at the same time is not possible. By contrast, (1) suggests that HS may become native-like, but do so rarely. I find it question- 
able whether end states should be part of the definition of HS. After all, we do not define second language learners in terms of incomplete acquisition either.

Second, as mentioned above, it is not so clear whether simultaneous bilinguals are part of the group of HS or not. According to (3) they are, according to (2) they might be, while Valdés' definition in (4) excludes simultaneous bilinguals. By definition, simultaneous (2L1) speakers have two first languages. Consequently, in (3), both heritage language and majority language should have been labeled as L1s. The only possible scenario according to (4) seems to be that speakers heard only one language at home; thus, the contact with their two languages happened successively. Therefore, some clarification is needed as to what extent BMP agree with Valdéz (or not).

Finally, based on these descriptions of HS, it does not become sufficiently clear (to me) in what respect HS are not, or not only, speakers of minority languages who divide into simultaneous and successive bilinguals. The only difference I can gather from BMPs text is related to the assumption that HS often acquire their languages incompletely. All in all, further clarification is needed as to how exactly, if at all, heritage speakers differ from simultaneous or successive speakers of a minority language. Given that (4) is introduced as Valdés' definition, I take (1) through (3) to be BMPs view of HS, and I assume that simultaneous bilinguals are not excluded from this population.

Finally, I suspect that, for many researchers, individuals commonly referred to as "heritage speakers", e.g., a Korean HS or a Spanish HS in the U.S., are 100\% minority in terms of ethnic/racial heritage. Put differently, both parents, typically immigrants, share the same heritage. However, this is not explicitly stated in any of BMPs descriptions. In the following discussion, I will therefore also include data from speakers who are 50\% minority (i.e. have only one parent who speaks the minority language).

\section{A concept that is poorly understood outside of the U.S.?}

BMPs section 2 begins by stating that "[t]he terms heritage language and heritage speaker are fairly new, and they are still poorly understood outside of North America, where similar concepts are denoted by the phrases minority language/ speaker" (p. 132).

First, as mentioned above, I take further clarification to be required in what respect minority language/speakers are different from heritage speakers (except 
in supposedly acquiring languages "incompletely" or in a divergent fashion). Second, the above statement is far from true.

As is well known, Europeans have been in contact for several hundreds of years. Presently, Europe's 10.5 million square kilometers cover 50 countries and more than 100 languages. European languages, moreover, display an impressive variety including the Romance, Germanic, Slavic, Baltic, Turkic, Celtic, Kartvelian and Semitic families, as well as Armenian, Albanian, Greek and Basque. Despite the great variety, the number of official languages amounts to only 24 . This means that there are many minority languages (and speakers). Since multilingualism and the protection of regional and minority languages are recognized political goals in present day Europe, many of these speakers are likely to actively use and transmit these languages to the next generation, thus protecting their linguistic heritage.

BMP provide no evidence of having attempted to relate the findings from child bilingualism to their own research agenda, although an impressive number of studies have been conducted in Europe ever since the late 1980s, and even earlier. By and large, these studies show that bilingual children are capable of separating their two languages from early on (e.g., Meisel 1989, De Houwer 1990 as well as the overviews in de Houwer 1995 and Meisel 2001, 2004). If these studies are externally valid, then bilingual children typically pass through the same acquisition stages as monolingual children, and they are subject to the same type of developmental errors. Not all, but most of these children match the definitions (2) and (3) of HS in one of their languages. ${ }^{1}$ Nevertheless, they appear to be acquiring a minority language successfully, in stark contrast to the stereotypical HS depicted by BMP. Thus, the question emerges how and why the acquisition outcome, according to BMP, is often incomplete although monolinguals and bilinguals acquire most aspects of syntax and morphology very early in life. Of course, one could discount studies with, e.g., German-French, SwedishItalian or Dutch-English bilingual children on the grounds that these are not HS, but one would then still have to explain how they differ from HS. Or, if they do match the concept of HS, one might turn to a more interesting question: is it possible that around school age, when many children start attending monolingual schools in the majority language, certain linguistic properties are not yet part of their internalized knowledge, even though witnessed in their speech?

In my view, the fact that the term "heritage speaker" has not been used in Europe - at least not until recently - should not be taken to imply that Europeans

1 Children who grow up in bilingual regions or countries with two official languages, e.g. in Québec or Switzerland, could be excluded in that, officially, there is no minority language - although whether in fact this is so may be debated. 
only have a vague idea of that concept. At any rate, such statements fail to convince as long as BMP do not clarify exactly what might distinguish HS from the speakers which most European-based researchers call simultaneous or successive bilinguals in their minority/weaker language.

\section{How typical is non native-like attainment?}

According to (1), HS are more likely to end up with non-native linguistic skills than with the linguistic skills of monolingual speakers. For instance, people who grow up bilingually, say in the U.S., with Portuguese and English, will normally not acquire the same competence as monolingual speakers growing up in Portugal or Brazil. But it is less clear if this observation amounts to more than a triviality. Polinsky and Keagan (2007) have already discussed the problem of the appropriate "baseline", suggesting comparisons with first generation immigrants. Nevertheless, many studies continue to compare HS to monolinguals.

As BMP discuss in section 5, differences in proficiency among HS, as well as between HS and monolingual speakers, may have a variety of causes. For example, HS may be subject to language input from the first generation which has already undergone changes under the influence of the (L2) majority language. HS receive massive input from the majority language both when acquiring the language and while using it as adults. Most importantly, they normally lack formal training in the language. The preconditions for language acquisition, therefore, are very different from those of a person who grew up using only one language at home, a person whose only language coincided with that of the larger society and was also the language of instruction at school.

In the following, I argue that reduced input alone cannot explain why second generation HS do not always sound like monolinguals (although I have no doubts that "interrupted" input may explain this). In fact, the linguistic skills of HS look far better than "incomplete" once one controls for crucial variables.

First, in terms of methodology, one possibility (illustrated further below) is to compare HS to bilingual ${ }^{2}$ speakers with the same language combination. Instead of comparing bilingual Italian speakers in Germany to monolingual Italian speakers in Italy, for instance, one should compare them to bilingual Italian speakers in Italy, thus minimizing potential effects of dual vs. exclusive language input.

2 I use the term bilinguals in the sense of early bilingual, and thus in opposition to L2 speaker. 
Second, whether or not non-native attainment is typical of heritage speakers largely depends on the population studied. To minimize potential effects of lack of schooling, for instance, one could include speakers who had schooling in their heritage language during childhood, rather than treating them as exceptions. As outlined below, a rather different picture of HS emerges if one studies HS who have received schooling in the heritage languages and instruction in more formal registers.

In a series of studies, Kupisch, Lein, Barton, Schröder, Stangen and Stöhr (2013) and Kupisch, Akpinar \& Stöhr (2013) have investigated the French of 11 bilinguals who grew up predominantly in Germany with one German-speaking and one French-speaking parent. These bilinguals meet BMPs descriptions of heritage speakers in (2) and (3). Some of them had attended a French school (the Lycée Français de Hambourg), where all instruction was in French, although they tended to speak German outside the classroom. Most travelled to France on a regular basis, but only three had been living in Francophone countries for several years. At the time of testing, they were between 20-42 years old. All but one preferred speaking German to speaking French, and they were more proficient in German according to a cloze test. Instead of comparing them to monolingual French speakers, they were compared to bilinguals who also acquired German and French simultaneously, but grew up predominantly in France.

These bilingual speakers of French were studied with respect to several properties where German and French differ, including adjective placement, article use in DPs, gender assignment and agreement, choice of prepositions, and voice onset time (VOT). Except for adjective placement, the HS of French did not significantly differ from the control group who grew up predominantly in France. The HS produced a total of 2010 prepositions in their spontaneous speech, and only 35 (1.7\%) were used inappropriately. In both an elicited production task and in a acceptability judgment task, the HS were 95\% correct in marking gender agreement (Kupisch, Akpinar and Stöhr 2013). In a task where speakers had to correct bare nouns into DPs with articles, they succeeded in providing the article $96 \%$ of the time - although in a comparable task the HS studied by Montrul and Ionin (2010, 2012) and the Italian HS in Kupisch (2012) encountered major difficulties.

Despite the absence of significant differences between the French HS and the majority speakers of French, minor differences in performance between the two groups could be observed. First, the HS were somewhat less accurate in gender assignment than in gender agreement, while the bilinguals who grew up in France showed no such difference. Second, HS' placement of adjectives was less accurate when placement was dependent on certain lexical properties of the adjectives, while the bilinguals who grew up in France were equally successful with 
all types of adjectives. Third, the heritage speakers' VOT values for / $\mathrm{t} /$ and / $\mathrm{k} / \mathrm{dif}-$ fered significantly from those of monolinguals reported in the literature, while those of the majority speakers of French showed no significant difference from monolingual values. Finally, when the HS' accent was judged by 23 monolingual French-speakers, most of them were deemed foreign. In summary, there were minor differences from French-dominant bilinguals related to lexical knowledge, and major differences related to accent, but no differences in their morphosyntactic abilities.

These findings serve to relativize BMPs claims about incomplete and divergent acquisition. These French-speaking bilinguals match the descriptions of HS in (1-3) in French. They were unbalanced bilinguals, who felt more comfortable using German than using French and were more proficient in German. However, they may have had advantages over other HS because they had received schooling in French as well as the occasion to spend time in French-speaking countries. ${ }^{3}$ The example shows that, under particular conditions - specifically with consistent input and instruction at school -, HS can become indistinguishable from native-speakers in many aspects of their language, although they have had relatively less input and featured another language which is stronger. They thus represent HS at the higher end of the proficiency scale. Notice that these speakers meet BMPs concept of HS in that the lexicon appeared to be one of the most vulnerable areas. However, as I discuss below, their accent was clearly the weakest aspect of their language, although BMP claim that accent is well preserved in HS (p. 136ff).

\section{Are simultaneous bilinguals more "at risk"?}

BMP report that in some specific structures the difference between a simultaneous and a sequential bilingual affects the quality of the heritage grammar (p. 134). BMP refer to a study by Montrul (2002), showing that simultaneous bilingual HS were more inaccurate at judging the meaning of the preterite and imperfect in Spanish than sequential bilingual HS. According to Montrul (2008), language attrition in childhood is more severe in simultaneous bilinguals.

If language attrition occurs within early (pre-puberty) bilingualism, it will be more severe in simultaneous bilinguals (exposed to the two languages very early) than in sequential bilinguals (when the L1 was acquired before the L2). (Montrul 2008, p. 60)

3 On the other hand, HS who attend language classes in the heritage language at the university do not appear to be a priori excluded in studies on heritage language acquisition. 
This assumption is perhaps intuitively plausible, because simultaneous bilinguals are confronted with majority language-input from the earliest possible moment in language development, thus leaving less space for the heritage language to develop. However, one should ask to what extent this assumption is valid, because adopting it uncritically may have an adverse effect on parents who are going to raise their children bilingually, as well as on educators who advise them.

Montrul's above claim appears to contradict studies on child bilingualism, in which the one parent - one language strategy has been identified as an appropriate method when raising children bilingually. Many studies have shown that simultaneous bilinguals can successfully acquire linguistic properties in both of their languages, even if these properties differ (see references above). ${ }^{4}$

Studies with adult simultaneous bilinguals from families where both languages are spoken are welcome on the grounds that they allow for an exact determination of age of onset (AoO) in both languages. For successive bilinguals, it is comparatively more difficult to say when exactly they have been exposed to the majority language for the first time. On the one hand, they are continuously surrounded by majority language speakers and they normally have access to the media in the majority language. On the other hand, it is rather unclear when they start to be interested in stimuli that are not directed to them personally.

The aforementioned studies by Kupisch et al. (forthcoming) and Kupisch, Akpinar and Stöhr (2013), focusing on French-German simultaneous bilinguals, demonstrated that many properties are acquired successfully, even if these differ in the bilinguals' two native languages. These studies can be taken to imply that reduced input during childhood alone does not lead to incomplete acquisition or divergence. One may argue, however, that the French-German bilinguals referred to above were only $50 \%$ heritage in the sense that only one of their parents represented the heritage language. Can we provide additional evidence from bilinguals who are $100 \%$ heritage, showing that simultaneous bilinguals are not necessarily at a disadvantage?

Kupisch, Akpinar, Belikova, Özçelik, Stangen and White (in prep.) investigated a population of 21 Turkish heritage speakers in Germany. Both parents were native speakers of Turkish. Participants predominantly used Turkish at home, but they considered German to be the language in which they were more proficient in terms of reading, writing, speaking and understanding. The group included 11 2L1ers whose first intensive contact (AoO) with German was between 0-3 years and 10 early L2-learners (AoO 4-7 years).

4 Of course, this does not hold if input in one of the languages is insufficient (see, e.g., Schlyter and Håkansson 1994 for such a case) 
The study tested knowledge of the definiteness effect (DE) in both languages by means of an oral acceptability judgment task. German shows the DE in positive and negative existentials (5a,c), while Turkish shows the DE in positive existentials (5b) but not in negative existentials ( $5 d)$ :

(5) a. ${ }^{*}$ Es ist der $/{ }^{*}$ es gibt den Hund in meinem Garten.
it is the/ it gives the dog in my garden

'There is the dog in my garden.'

b. ${ }^{*}$ Bahçe-de her çocuk var. garden-loc every child exist

'There is every child in the garden.'

c. ${ }^{\star E S}$ ist nicht der/*es gibt nicht den Hund in meinem Garten. it is the/ it gives the dog in my garden

'There is the dog in my garden.'

d. Bahçe-de Ali yok.

garden-Loc Ali not-exist

'There isn't Ali in the garden.'

Despite the differences in negative existentials between German and Turkish, HS of Turkish did not perform significantly differently from monolingual controls. In German, there were no significant differences between the bilinguals and the monolinguals. In Turkish, the speakers performed on a par with monolinguals except in one condition which included negative quantifiers. Crucially, all bilinguals treated German and Turkish existentials differently. In the condition with negative quantifiers the difference between monolinguals and bilinguals was due to the early L2-speakers performing differently, although they had been exposed to German later than the other group. This result contradicts the assumption that simultaneous bilinguals are at a disadvantage. The results further suggest that reduced input during early life does not necessarily lead to incomplete or divergent acquisition.

\section{Phonetic and phonological skills}

According to BMP, "phonological competence seems to be the best-preserved aspect of linguistic knowledge in heritage speakers, although it is not entirely nativelike" (p. 136).

Phonology, in general, seems to be the best-preserved area of heritage grammar, followed by syntax, while inflectional morphology, semantics, and the syntax-discourse interface are the most vulnerable. (p. 153) 
The major evidence for this assumption seems to be that low proficiency speakers have unimpeded phoneme perception, and outperform L2 learners' phonological abilities, as reported in section 3.1.2. However, the report does not specify whether linguistic skills other than phonetic and phonological abilities have been systematically investigated in the same group of HS, or whether they are simply assumed based on self-reports. In the following, I would like to add some data to the picture. These data show that if one compares productive phonetic and phonological skills and morpho-syntactic properties (including the interfaces), the former will be more deviant from the monolingual (and bilingual majority language) baseline.

I reported above that the VOT values produced by HS of French studies in Kupisch et al. (forthcoming) differed significantly from the values reported for monolingual French speakers. In addition, when the same heritage speakers of French were rated by monolingual speakers in terms of their foreign accent, they were deemed significantly more often as foreign than the French majority speakers. Overall, the group of HS was rated native $49 \%$ of the time. On the individual level, only two out of the ten speakers were rated native more than $80 \%$ of the time. By contrast, the majority language speakers of French were rated native $90 \%$ of the time on average, while on the individual level 8 out of 10 speakers were deemed native.

Additional evidence that pronunciation is particularly vulnerable has been provided in a series of studies with 10 heritage speakers of Italian with German as their other first language. Again, these bilinguals grew up in bi-national families and learnt Italian mostly in the home context. Most of them travelled to Italy on a regular basis (normally for summer vacations). Unlike the French heritage speakers referred to above, they attended monolingual German schools. At the time of testing, they were between 19 and 39 years old, preferred speaking German to speaking Italian, and were more proficient in German according to a cloze test. Again, they were not compared to monolingual speakers, but to bilingual speakers with the same language combination who spoke Italian as a majority language. It was shown that the heritage speakers performed rather well (above $92.8 \%$ accuracy) with respect to gender agreement on past participles (Bianchi 2013: 12), while they performed somewhat less accurately (79.7\%) with regard to gender assignment (Bianchi 2013: 12) and adjective placement (82.6\%) (Kupisch 2013). Moreover, they had noticeable problems correcting bare nouns to DPs with definite articles in generic DPs (accuracy 33\%), where these were required in Italian but not in German (Kupisch 2012). The same speakers were assessed in terms of global foreign accent by 20 monolingual raters of Italian (Kupisch, Barton, Hailer, Lein, Stangen and van de Weijer, in prep.). As a group, they were deemed native only $30 \%$ of the time. On the individual 
level, only two out of ten speakers were taken to be native more than $80 \%$ of the time.

In summary, these results indicate that phonetic and phonological properties are relatively problematic in HS, which appears to run counter to BMPs findings. There are three possible explanations. First, HS' phonological skills may have been overrated by BMP because there were no direct comparisons of phonological abilities and other aspects of their language in the same group of speakers. Second, the raters in the accent studies (summarized above) may have paid attention to cues beyond pronunciation, e.g. choice of words or idiomaticity, which would mean that their ratings were based on properties beyond perceived accent. Third, the relative vulnerability across linguistic domains may differ between HS at the higher end of the proficiency scale and HS at the lower end of the proficiency scale.

\section{Conclusions}

In this contribution, I have looked at the concept of HS from a European perspective, foregrounding bilinguals at the higher level of the proficiency scale. I have argued that there is no good reason for excluding highly proficient bilinguals, whether simultaneous or successive, from research on HS, as long as they match current descriptions of HS in the literature. I have pointed out further that it is not clear in what respect, or if at all, HS differ from bilingual speakers of a minority language. I have summarized recent studies on $\mathrm{HS}$ at the higher level of the proficiency scale, showing that, under particular conditions, such as schooling in the heritage language, HS can become indistinguishable from majority language speakers and monolinguals, although their relative input was reduced in both languages throughout their lifespan. Finally, I have shown that the phonology of HS appears to be the most vulnerable area, contrary to BMPs findings.

\section{References}

Bianchi, Giulia. 2013. Gender in Italian-German Bilinguals: a comparison with German L2 learners of Italian. Bilingualism: Language and Cognition 16(3). 538-557.

De Houwer, Annick. 1990. The acquisition of two languages from birth: A case study. Cambridge, UK: Cambridge University Press.

De Houwer, Annick. 1995. Bilingual language acquisition. In P. Fletcher \& B. MacWhinney (eds.), Handbook of Child Language, 219-250. London: Blackwell. 
Kupisch, Tanja. 2012. Generic subjects in the Italian of early German-Italian bilinguals and German learners of Italian as a second language. Bilingualism: Language and Cognition 15(4). 736-756.

Kupisch, Tanja. 2013. Adjective placement in simultaneous bilinguals (German-Italian) and the concept of cross-linguistic overcorrection. Bilingualism: Language and Cognition. Forthcoming.

Kupisch, Tanja, Alyona Belikova, Öner Özçelik, Ilse Stangen \& Lydia White. 2013. On complete acquisition in heritage speakers: The definiteness effect in German-Turkish bilinguals. Ms., Lund/McGill/Indiana/Hamburg.

Kupisch, Tanja, Dagmar Barton, Katja Hailer, Tatjana Lein, Ilse Stangen \& Joost van de Weijer. in prep. Foreign accent in adult simultaneous bilinguals. Ms., University of Hamburg/Lund University. Submitted to Heritage Language Journal.

Kupisch, Tanja, Dagmar Barton, Tatjana Lein, D. Judith Schröder, Ilse Stangen \& Antje Stöhr. forthcoming. Acquisition outcomes across domain in adult heritage speakers of French. Journal of French Language Studies.

Kupisch, Tanja, Deniz Akpinar \& Antje Stöhr. 2013. Gender assignment and gender agreement in adult bilingual and second language speakers of French. Linguistic Approaches to Bilingualism 3(2). 150-179.

Meisel, Jürgen M. 1989. Early differentiation of languages in bilingual children. In K. Hyltenstam and L. Obler (eds.), Bilingualism Across the Lifespan: Aspects of Acquisition, Maturity, and Loss, pp. 13-40. Cambridge, UK: Cambridge University Press.

Meisel, Jürgen M. 1994 (ed). Bilingual First Language Acquisition: French and German Grammatical Development. Amsterdam: John Benjamins.

Meisel, Jürgen M. 2001. The Simultaneous Acquisition of Two First Languages: Early Differentiation and Subsequent Development of Grammars. In J. Cenoz \& F. Genesee (eds), Trends in Bilingual Acquisition, 11-41. Amsterdam: John Benjamins.

Meisel, Jürgen M. 2004. The Bilingual Child. In T.K. Bhatia \& W.C. Ritchie (eds), The Handbook of Bilingualism (Blackwell Handbooks in Linguistics), 91-113. Oxford: Blackwell Publishers.

Montrul, Silvina \& Tania Ionin. 2010. Transfer effects in the interpretation of definite articles by Spanish heritage speakers. Bilingualism: Language and Cognition 13(4), 449-473.

Montrul, Silvina. 2002. Incomplete acquisition and attrition of Spanish tense/aspect distinctions in adult bilinguals. Bilingualism: Language and Cognition 5, 39-68.

Montrul, Silvina. 2008. Incomplete acquisition in bilingualism: Re-examining the age factor. Amsterdam: John Benjamins.

Polinsky, Masha \& Olga Kagan. 2007. Heritage languages: In the 'wild' and in the classroom. Language and Linguistics Compass 1, 368-395.

Schlyter, Suzanne \& Gisela Håkansson. 1994. Word order in Swedish as the first language, second language and weaker language in bilinguals. Scandinavian Working Papers on Bilingualism 9, 49-66. 